\title{
Phenomenological MSSM interpretation of CMS results
}

\author{
Samuel Bein** \\ CMS collaboration \\ E-mail: samuel.bein@cern.ch
}

Using a global Bayesian analysis, it is shown how the results from 7 and $8 \mathrm{TeV}$ searches for supersymmetry performed by the CMS experiment constrain the Minimal Supersymmetric Standard Model (MSSM). The study is performed within the framework of the phenomenological MSSM (pMSSM), a 19-parameter realization of the R-parity conserving weakscale MSSM, that captures most of the latter's phenomenological features and which, therefore, permits robust conclusions to be drawn about the MSSM.

38th International Conference on High Energy Physics 3-10 August 2016

Chicago, USA

\footnotetext{
* Speaker.

${ }^{\dagger}$ Thanks to the organizers for putting on a wonderful conference.
} 


\section{Introduction}

Searches [1-11] for new physics at CMS [12] during Run 1 have placed stringent limits on the masses of supersymmetric (SUSY) particles [13-18]. However, these limits are usually derived under the assumption that the SUSY particle mass spectrum resembles one of a set of simplified models [19-21]. Because this set does not cover the entire range of experimentally possible outcomes allowed by the minimal supersymmetric standard model (MSSM), it is informative to perform a global interpretation of searches in terms of a model that better covers the MSSM to assess the viability of the weak-scale SUSY hypothesis.

An analysis [23] of the viability of a potentially discoverable subset of the MSSM is presented in the context of a SUSY model who's range of allowed phenomena is more equivalent to that of the full MSSM compared with simplified models. The model, called the phenomenological MSSM (pMSSM) [22], is a submodel of the MSSM that makes a minimal number of assumptions about the MSSM, namely that

- there are no new flavor changing neutral currents;

- there are no new sources of $\mathrm{CP}$ violation at tree level;

- the first and second generation sfermion (scalar fermion) masses are degenerate, and

- the lightest of the SUSY particles (sparticles) is the neutralino.

These assumptions reduce the dimensionality of the MSSM from 111 free parameters to 19 pMSSM parameters, which are:

- three gaugino mass parameters $M_{1}, M_{2}$, and $M_{3}$;

- one higgsino mass parameter $\mu$ and one pseudo-scalar Higgs mass $m_{A}$;

- ten sfermion mass parameters $m_{\tilde{f}}$, where $\tilde{f}=\tilde{Q}_{1}, \tilde{U}_{1}, \tilde{D}_{1}, \tilde{L}_{1}, \tilde{Q}_{3}, \tilde{U}_{3}, \tilde{D}_{3}, \tilde{L}_{1}$, and $\tilde{E}_{3}$ (imposing $\left.m_{\tilde{Q} 1}=m_{\tilde{Q} 2}=m_{\tilde{L} 1}=m_{\tilde{L} 2}\right)$;

- three trilinear couplings $A_{t}, A_{b}$, and $A_{\tau}$, and

- the ratio of the Higgs vacuum expectation values $\tan \beta=v_{2} / v_{1}$.

An interpretation of a representative set of 7 and $8 \mathrm{TeV}$ CMS SUSY searches is performed using a Bayesian analysis, where a posterior density is constructed based on 1) a prior probability density that depends on low-energy, non-direct CMS (non-DCS) constraints, and 2) a likelihood that takes into account the CMS results:

$$
p\left(\theta \mid D^{\mathrm{CMS}}\right) \propto L\left(D^{\mathrm{CMS}} \mid \theta\right) p^{\text {non-DCS }}(\theta),
$$

where $\theta$ refers to a point in the pMSSM parameter space, and $D^{\mathrm{CMS}}$ refers to the data observed by CMS.

The prior is constructed as the density of points in a Markov chain Monte Carlo scan of the 19 pMSSM parameters. In the scan, the parameters are allowed to vary, subject to the non-DCS 
constraints, within a subset of the pMSSM that is potentially discoverable at the LHC, namely, within the SUSY particle mass range between 0 and $3 \mathrm{TeV}$ and trilinear couplings between -7 and $7 \mathrm{TeV}$. The ur-prior, that is, the probability density before the incorporation of any constraints, is taken to be uniform in the space of the 19 pMSSM parameters. Due to limitations of the detector simulation software, pMSSM points featuring long-lived charginos were not considered. The likelihood assumes various forms, depending on the analysis considered. Most searches employ a counts likelihood based on the bin-by-bin observed and expected counts in all or most signal regions of the corresponding analysis, while others are based on $\chi^{2}$ values derived from a global fit performed accross all bins of an analyses. Further details on the construction of the prior and likelihoods are given in [23].

This installment of the proceedings highlights some key findings of [23], including how the 7 and $8 \mathrm{TeV}$ CMS results constrain the MSSM, and sheds light on model points that evaded the CMS analyses, despite their having large SUSY production cross sections.

\section{Constraints on sparticle masses}

The impact of CMS searches is perhaps best seen by comparing the prior and posterior probability densities (Eq. 1.1) as a function of pMSSM parameters. Three such comparisons are shown for densities projected onto the dimensions of the gluino, LSP, and squark masses in Fig. 1. The black solid histograms represent the posterior density corresponding to the combined constraints of all considered 7 and $8 \mathrm{TeV}$ CMS searches. In all three cases, the low-mass regions are heavily suppressed in favor of higher mass regions, reflecting the lack of evidence for SUSY in the data observed by the considered searches. Masses of the color-charged particles are excluded below about $400 \mathrm{GeV}$, and the most probable values of these parameters are pushed higher by about 500 $\mathrm{GeV}(1 \mathrm{TeV}$ ) for squarks (gluinos). No lower bound can be placed on the LSP mass, since the posterior density has a substantially nonzero value in the lowest LSP mass bin.

A projection of the posterior density onto the plane of the gluino and LSP masses is given in Fig. 2, along with the survival probability. The survival probability is defined as the fraction of considered pMSSM points that remain non-excluded at a significance level of $2 \sigma$, after the constraints of the Run 1 searches. The most probable region features gluino and LSP masses of 3 $\mathrm{TeV}$ and $300 \mathrm{GeV}$, respectively. The survival probability indicates that the CMS searches exclude scenarios with $3 \mathrm{TeV}$ gluino masses at a rate of about 50\%, for small LSP mass. This surprisingly high sensitivity to large gluino masses is attributable primarily to the presence of other (non-gluino) sparticles with significant production cross sections in the models with heavy gluinos, and to the increase in the squark production cross section that is a consequence of the presence of heavy gluinos that mediate squark production ( $t$-channel squark production with gluino propagators).

\section{Non-excluded points}

An interesting set of physics processes characterize the region of the pMSSM close to the diagonal of the $m(\tilde{g})-m\left(\tilde{\chi}_{1}^{0}\right)$ plane. Many models in this region feature cascade decays involving other sparticles, frequently electroweakinos (EW-kinos), with small mass splittings between all involved particles. The small mass splitting results in low- $p_{\mathrm{T}}$ decay products and, in general, leads 

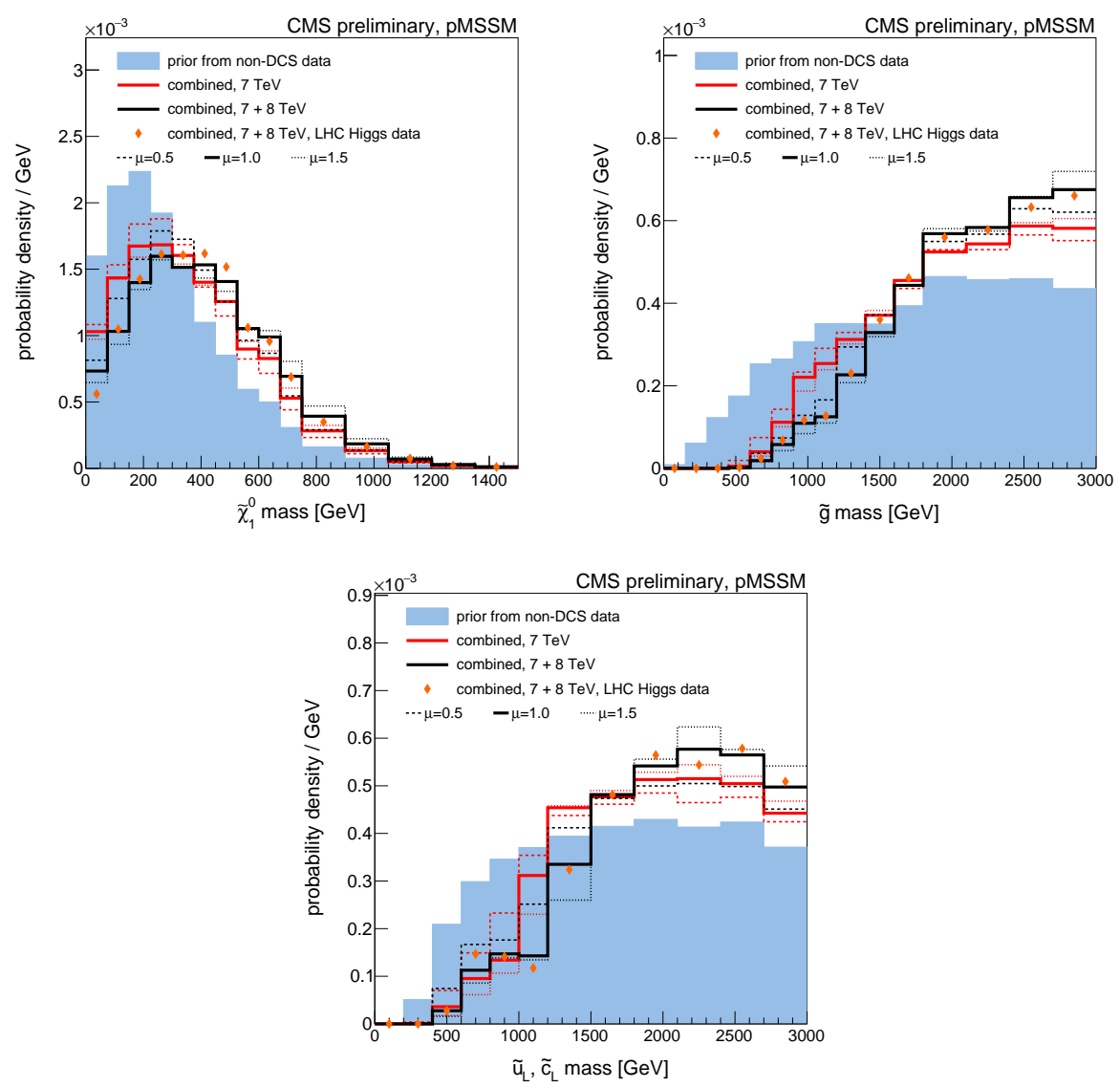

Figure 1: The probability density of sparticle masses without (shaded blue histograms) and with (solid line histograms) the constraints of CMS Run 1 SUSY searches [23].

to events with low acceptance. The events often populate regions of kinematic phase space that are dominated by standard model backgrounds, such as the low- $H_{\mathrm{T}}$ and low- $p_{\mathrm{T}}^{\text {miss }}$ regions.

Because of the lowered sensitivity of searches to points in the diagonal region, small gluino masses survive at an increased rate near the diagonal. The surviving pMSSM points with the smallest gluino mass fall in this region. Diagrams depicting the principal physics processes associated with two of these points are shown in Fig. 3. In both cases the mass splitting between the gluino and LSP is less than $100 \mathrm{GeV}$, and the mass splitting between the intermediate EW-kino and LSP is less than $5 \mathrm{GeV}$. The latter mass splitting is not small enough to result in long- or medium- lived charginos, but it is sufficiently small to render the decay products of the standard model bosons too soft to satisfy object selection requirements of most analyses. Special analysis techniques may be required to target the types of processes in Fig. 3 specifically. Importantly, while the diagrams themselves can be found among the set of simplified models used to design LHC SUSY searches, the feature of the low mass splitting between the intermediate EW-kino and the LSP is not typically considered, and this makes a considerable difference to the characteristics of the signal events.

To complicate matters, the trigger acceptance for the signal events of these model points is 

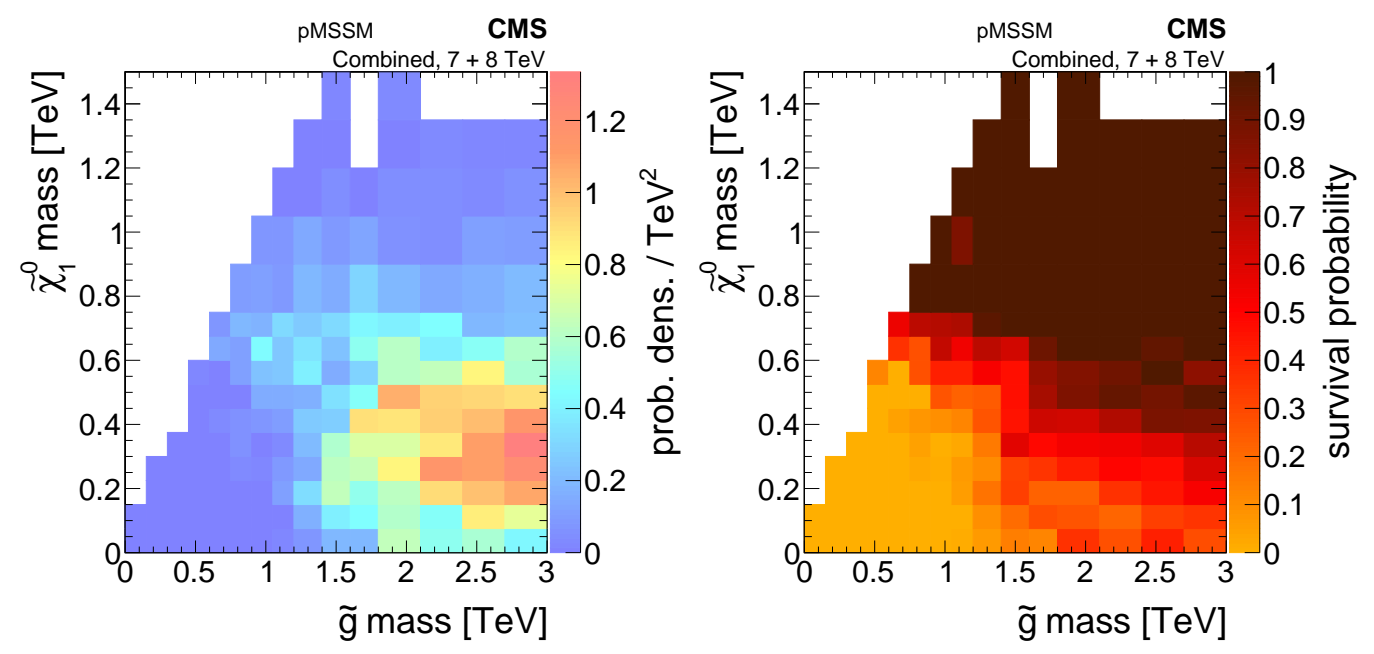

Figure 2: The probability density (left) and survival probability of the pMSSM model space as a function of the gluino and LSP masses, after the Run 1 CMS constraints [23].
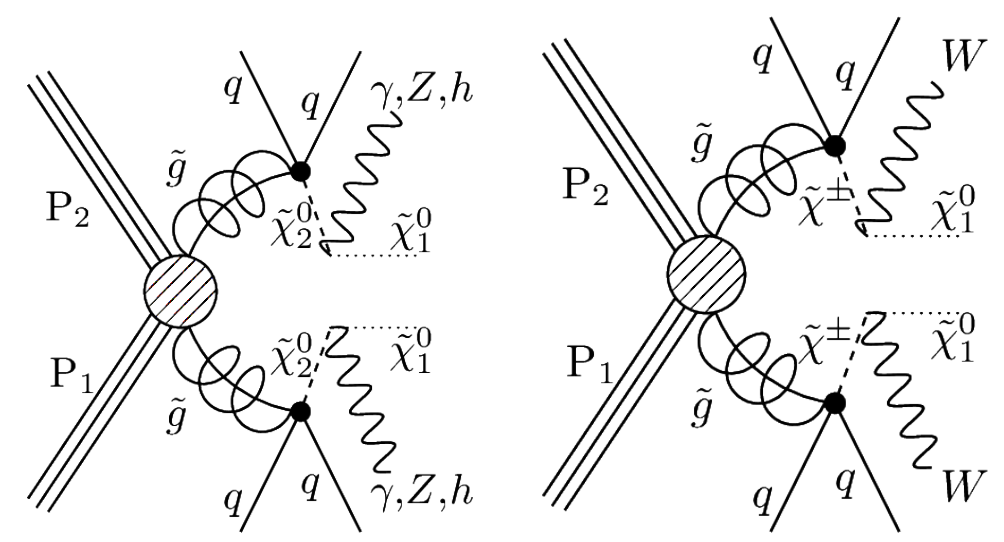

Figure 3: Principal processes characterizing the pMSSM points with the lowest gluino masses that survived the CMS Run 1 analyses. The gluino masses are on the order of $600 \mathrm{GeV}$, and the mass difference between the gluino and LSP is around $100 \mathrm{GeV}$.

rather low, at least for triggers that rely only on a $p_{\mathrm{T}}^{\text {miss }}$-like observable. To see why, sketches of the $H_{\mathrm{T}}$ and $H_{\mathrm{T}}^{\text {miss }}\left(H_{\mathrm{T}}^{\text {miss }}\right.$ is a $p_{\mathrm{T}}^{\text {miss }}$ proxy) distributions are shown for the two lightest gluino pMSSM points in Fig. 4. Survivor 1 refers to the PMSSM point characterized by the right diagram in Fig. 3. and survivor 2 refers to the point characterized by the left diagram. With survivor 1 (blue), the majority of the $H_{\mathrm{T}}^{\text {miss }}$ distribution resides below the saturation level of a typical $p_{\mathrm{T}}^{\text {miss }}$ trigger (approximately $200 \mathrm{GeV}$ ), and with survivor 2 (green), nearly $100 \%$ of the events lie below this threshold.

A possible strategy for probing survivor 1 would be to consider events with small but nonzero $p_{\mathrm{T}}^{\mathrm{miss}}$, with one or two non-isolated leptons whose trajectories are somewhat collinear with the $p_{\mathrm{T}}^{\text {miss }}$ vector. This would isolate events that have initial state radiation boosting the final state 

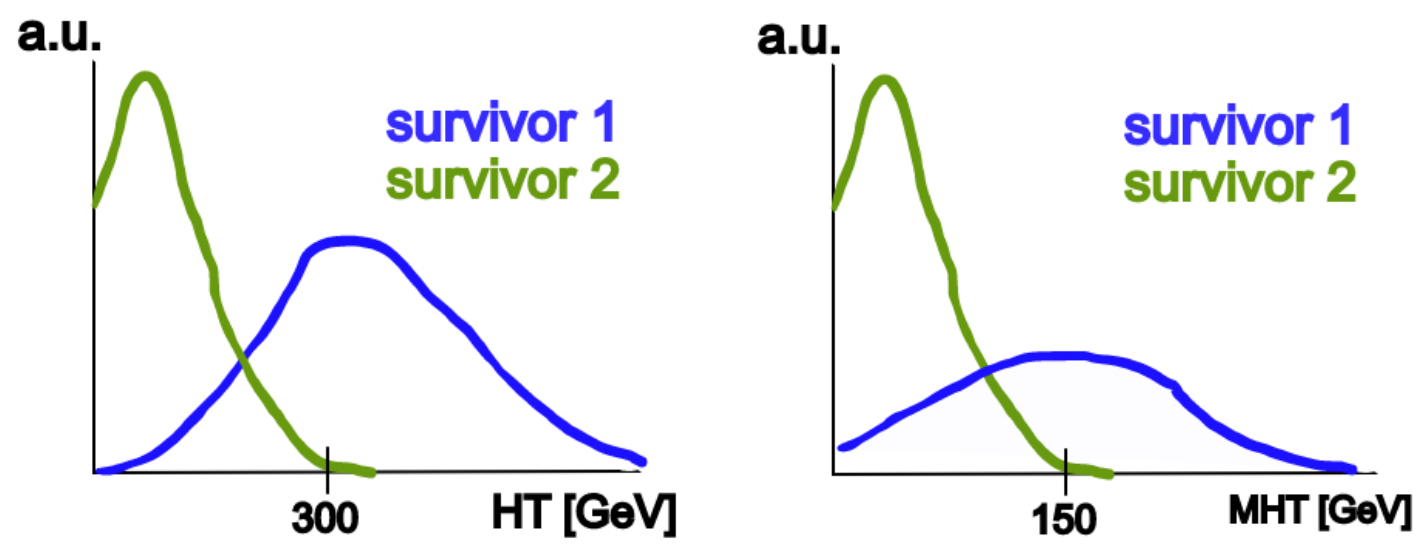

Figure 4: Sketches of the distributions of key observables corresponding to the right-hand diagram (survivor 1) and left-hand diagram (survivor 2) shown in Fig. 3.

particles into the direction of the $p_{\mathrm{T}}^{\text {miss }}$. A trigger with a similar set of selection would be ideal for maximizing signal acceptance. Strategies for probing the survivor 2 model are less certain, but a similar approach of selecting events containing tags of the non-isolated soft decay products, in this case photons or leptons, that are collinear with the $p_{\mathrm{T}}^{\text {miss }}$ vector, may prove effective.

\section{Summary}

A subset of the findings in [23] has been presented. In the context of the phenomenological MSSM, sparticles with color charge have been ruled out below about $400 \mathrm{GeV}$, and the probability density of the LSP mass has been shifted to significantly higher values, as a result of a set of Run 1 CMS SUSY searches. The searches were able to probe about $50 \%$ models with gluino masses up to $3 \mathrm{TeV}$, assuming an LSP mass less than $400 \mathrm{GeV}$. The two pMSSM points with the lowest gluino masses that survived the Run 1 searches correspond to signal events featuring gluino production with cascade decays involving EW-kinos, where the EW-kinos have mass splittings with the LSP, of less than $5 \mathrm{GeV}$. Techniques for probing these model points were discussed, and it was suggested that a search for signal candidate events with soft, non-isolated leptons or photons that have distinct topological relationships with the missing transverse momentum vector, may be be an effective way to achieve sensitivity to these challenging points.

\section{References}

[1] S. Chatrchyan et al. [CMS Collaboration], Phys. Rev. Lett. 109, 171803 (2012) doi:10.1103/PhysRevLett.109.171803 [arXiv:1207.1898 [hep-ex]].

[2] S. Chatrchyan et al. [CMS Collaboration], Phys. Rev. D 86, 072010 (2012) doi:10.1103/PhysRevD.86.072010 [arXiv:1208.4859 [hep-ex]]. 
[3] S. Chatrchyan et al. [CMS Collaboration], JHEP 1211, 147 (2012) doi:10.1007/JHEP11(2012)147 [arXiv:1209.6620 [hep-ex]].

[4] S. Chatrchyan et al. [CMS Collaboration], JHEP 1406, 055 (2014) doi:10.1007/JHEP06(2014)055 [arXiv:1402.4770 [hep-ex]].

[5] S. Chatrchyan et al. [CMS Collaboration], JHEP 1210, 018 (2012) doi:10.1007/JHEP10(2012)018 [arXiv:1207.1798 [hep-ex]].

[6] S. Chatrchyan et al. [CMS Collaboration], Phys. Lett. B 725, 243 (2013) doi:10.1016/j.physletb.2013.06.058 [arXiv:1305.2390 [hep-ex]].

[7] V. Khachatryan et al. [CMS Collaboration], Eur. Phys. J. C 75, no. 5, 235 (2015) doi:10.1140/epjc/s10052-015-3451-4 [arXiv:1408.3583 [hep-ex]].

[8] V. Khachatryan et al. [CMS Collaboration], JHEP 1506, 116 (2015) doi:10.1007/JHEP06(2015)116 [arXiv: 1503.08037 [hep-ex]].

[9] V. Khachatryan et al. [CMS Collaboration], JHEP 1504, 124 (2015) doi:10.1007/JHEP04(2015)124 [arXiv:1502.06031 [hep-ex]].

[10] S. Chatrchyan et al. [CMS Collaboration], JHEP 1401, 163 (2014) Erratum: [JHEP 1501, 014 (2015)] doi:10.1007/JHEP01(2015)014, 10.1007/JHEP01(2014)163 [arXiv:1311.6736, arXiv:1311.6736 [hep-ex]].

[11] V. Khachatryan et al. [CMS Collaboration], Eur. Phys. J. C 74, no. 9, 3036 (2014) doi:10.1140/epjc/s10052-014-3036-7 [arXiv:1405.7570 [hep-ex]].

[12] S. Chatrchyan et al. [CMS Collaboration], JINST 3, S08004 (2008). doi:10.1088/1748-0221/3/08/S08004

[13] P. Ramond, Phys. Rev. D 3, 2415 (1971). doi:10.1103/PhysRevD.3.2415

[14] Y. A. Golfand and E. P. Likhtman, JETP Lett. 13, 323 (1971) [Pisma Zh. Eksp. Teor. Fiz. 13, 452 (1971)].

[15] D. V. Volkov and V. P. Akulov, JETP Lett. 16, 438 (1972) [Pisma Zh. Eksp. Teor. Fiz. 16, 621 (1972)].

[16] J. Wess and B. Zumino, Nucl. Phys. B 70, 39 (1974). doi:10.1016/0550-3213(74)90355-1

[17] P. Fayet, Nucl. Phys. B 90, 104 (1975). doi:10.1016/0550-3213(75)90636-7

[18] D. J. H. Chung, L. L. Everett, G. L. Kane, S. F. King, J. D. Lykken and L. T. Wang, Phys. Rept. 407, 1 (2005) doi:10.1016/j.physrep.2004.08.032 [hep-ph/0312378].

[19] J. Alwall, P. Schuster and N. Toro, Phys. Rev. D 79, 075020 (2009) doi:10.1103/PhysRevD.79.075020 [arXiv:0810.3921 [hep-ph]].

[20] D. Alves et al. [LHC New Physics Working Group Collaboration], J. Phys. G 39, 105005 (2012) doi:10.1088/0954-3899/39/10/105005 [arXiv:1105.2838 [hep-ph]].

[21] S. Chatrchyan et al. [CMS Collaboration], Phys. Rev. D 88, no. 5, 052017 (2013) doi:10.1103/PhysRevD.88.052017 [arXiv:1301.2175 [hep-ex]].

[22] A. Djouadi et al. [MSSM Working Group Collaboration], hep-ph/9901246.

[23] V. Khachatryan et al. [CMS Collaboration], JHEP 1610, 129 (2016) doi:10.1007/JHEP10(2016)129 [arXiv:1606.03577 [hep-ex]]. 